\title{
Factors associated with knowledge of hypertension among adolescents: implications for preventive education programs in primary care
}

\author{
Iga Grad ${ }^{1}$, Agnieszka Mastalerz-Migas ${ }^{1,2}$ and Katarzyna Kiliś-Pstrusińska ${ }^{1,3^{*}}$
}

\begin{abstract}
Background: Hypertension (HT) amongst adolescents remains a vital issue of both a medical and social nature. There is a lack of data regarding the factors influencing the awareness of the disease among the youth. The aim of this study was to evaluate the knowledge about HT among adolescents and its level corresponding to the selected demographic, environmental and medical factors.

Methods: The study was carried out among 250 adolescents of secondary schools. The authors' questionnaire poll and the psychological tests Personal Values List (PVL) and Personal Competence Scale (PCS) were performed.

Results: Only 13.2\% of the youth surveyed displayed the "medium" level (defined below) of HT knowledge. Most of them present satisfactory knowledge about the causes of HT. The children from urban areas generally displayed better knowledge about HT than their peers from rural regions. Only the children who had had their blood pressure previously examined displayed good knowledge about HT. The most frequently indicated source of this knowledge was school; however, its level still remains low. There was no significant association between the level of global knowledge about HT and the feeling of one's own competences and considering the category "good health" an important personal value.

Conclusions: Knowledge about HT among adolescents remains unsatisfactory and random, which indicates the necessity for routine education in this field, especially as it applies to HT symptoms. It seems that the consideration of such elements as blood pressure measurement and family history of HT in education programs can improve their efficiency.
\end{abstract}

Keywords: Hypertension, Adolescents, Knowledge About Hypertension

\section{Background}

Hypertension (HT) is known as one of the most significant risk factors of atherosclerosis and cardiovascular diseases. HT prevalence in the worldwide adult population is estimated at 26\% [1]. HT is also one of the most urgent health problems among children and adolescents $[2,3]$. It has been assessed that HT is common in $3.5 \%$ of the population at developmental age [4]. The highest HT frequency is observed among older children, and boys are more prone to it than girls [5]. Puberty, obesity and coexisting metabolic disorders lead to the fact that

\footnotetext{
* Correspondence: katarzyna.kilis-pstrusinska@umed.wroc.pl

'Department of Nursing, Public Medical Academy, Opole, Poland

${ }^{3}$ Department of Pediatric Nephrology, Wroclaw Medical University, Wroclaw, Poland

Full list of author information is available at the end of the article
}

primary hypertension (PHT) becomes a dominant form of hypertension among adolescents [6]. PHT is more and more often recognized without any clinical symptoms [7]. Similarly to adults, primary hypertension among children corresponds to obesity and a family history of this disease [8-10]. Obesity, hypercholesterolemia, hypertension and habits contributing to the risk of cardiovascular diseases which have their roots in childhood, tend to continue into adulthood. Hence, any preventive actions against these diseases in adulthood will be far too late. This is why, from an early age, programs promoting physical activity, healthy eating habits and a non-smoking policy should be undertaken in order to prevent the aforementioned diseases [11-13], especially when considering the fact that more than $40 \%$ of children have 
complications connected with organ damage at the moment of PHT diagnosis [14]. Any preventive education program should be tailored to its recipients and their knowledge of the program's subject. It should also correspond to the recipients' value systems.

Taking all the evidence into consideration, we decided to carry out this study of the knowledge of HT among adolescents as well as of healthy behavior predictors in the developmental age population. The aim of our study was to obtain the answer to the following questions: a) What is the level of knowledge about HT among adolescents? b) Are selected factors such as demographic (stage of education, sex, place of living), environmental (school, family), medical (previous blood pressure measurements, a previous diagnosis of high blood pressure, family history of HT or other chronic diseases) and others related to the level of knowledge about HT among adolescents? c) Is there any relationship between the level of knowledge about HT among adolescents and factors such as the system of values or personal competence?

\section{Methods}

The study was performed among adolescents from south-western Poland, Opole province, between April and June 2013. First, three schools were randomly selected among the 27 schools of that region (number of adolescents 11,000). Next, 300 students were randomly selected and they were invited to the study. Written informed consent was obtained from all the students. In the case of students under 18, the agreement was also signed by their parents. The study was conducted in the school during classes. Finally 250 students completed all the tests and these results were analyzed. The data concerning the demographic aspects of the tested group is presented in Table 1.

The study was carried out on the basis of: the authors' questionnaire and the psychological tests: Personal

Table 1 Demographic data of the examined students

\begin{tabular}{lll}
\hline Parameter & & $\begin{array}{l}\text { Students } \\
\mathbf{n}=\mathbf{2 4 9}(\mathbf{1 0 0 \% )})\end{array}$ \\
\hline Sex, n (\%) & female & $144(57.8)$ \\
& male & $105(42.2)$ \\
Age, n (\%) & 16 years & $32(12.85)$ \\
& 17 years & $96(38.55)$ \\
& 18 years & $102(40.96)$ \\
Secondary school grade, $\mathrm{n}(\%)$ & 19 years & $19(7.63)$ \\
& I & $74(29.7)$ \\
& III & $133(53.4)$ \\
Inhabited, $\mathrm{n}(\%)$ & city (urban) & $98(39.7)$ \\
& countryside (rural) & $149(60.3)$ \\
\hline
\end{tabular}

Competence Scale (PCS) and Personal Values List (PVL). The study protocol adhered to the Declaration of Helsinki and was approved by the Ethics Committee of Wroclaw Medical University (nr KB-378/2012).

The questionnaire consisted of questions concerning personal data, hypertension (epidemiology, causes, symptoms, complications, ways of treatment, preventive strategies), previous blood pressure measurements and HT diagnosis, as well as a family history of HT and other chronic diseases (see Additional file 1). To check if the information about HT among young people is accurate, a point scale was employed. The correct responses were given 1 point, the incorrect ones -2 points, don't know responses or a lack of response were 0 points. The percentage ranges of were correct responses $75 \%$ and above, $74-50 \%$ and lower than $50 \%$, and these were defined as good, medium and unsatisfactory knowledge, respectively. The clarity and certainty of the survey questions were confirmed in a pilot study.

\section{The Personal Competence Scale}

The Personal Competence Scale (PCS) is designed to assess generally self-effectiveness as well as its components such as: a feeling of strength to start an action (subscale A) and prevail to continue it (subscale B). Each subscale includes 6 statements of both positive and negative meaning. The students surveyed assessed 6 of the statements applying to the fact of starting an action and 6 others applying to the frequency of given behaviors. They did it by means of choosing one out of 4 responses (respectively, yes, rather yes, rather no, and no in the former, and almost always, often, sometimes and never in the latter). Consequently, by taking them altogether, and then subscales A and B separately, the sum of the points referring to each of the responses was calculated (from 1 to 4 points, depending on how the response was formed). This made it possible to estimate a general outcome of a feeling of self-effectiveness (12-48 points) as well as the parameters of feeling strong and stubborn to complete the task (for each of 12-48 points). The raw scores of the test have been converted to sten scores 1-10 (a standard scoring system). Interpretation of the sten scores is as follows: 1-4 represents low, 5-6 medium and 7-10 high test results, respectively. To assess the feeling of strength and perseverance, higher scores go to a greater feeling of strength/perseverance and, on the other hand, lower scores refer to a lesser feeling of strength/perseverance. The PCS reliability for the total scale is 0.72 , for the subscales, 0.74 and 0.62 (Cronbach's alpha coefficient), respectively. Its theoretical accuracy ratio for total PCS is 0.43 , and for subscales, 0.53 and 0.38 [15].

\section{The Personal Values List (PVL)}

In the test, the study subjects selected 5 out of 9 symbols of happiness which they consider the most important to 
them. The most important symbol was given 5 points, whereas the least important one 1 point. Further on, the adolescents chose and determined the scores for 10 personal values, in accordance with the rule above. The symbols of happiness as well as personal values that were not chosen were given 0 points each. The rank assigned to "health" by the youth reflects the value which is given to "health" in the context of other individual values and possessions. The Personal Values List's reliability is 0.78 and 0.76 for both of the parts (test-retest). Its theoretical accuracy is 0.74 [15].

Statistical analysis was performed with an $\mathrm{R} 2.10 .1$ package (for Mac OS X Cocoa GUI). Two types of Chisquare were used, compliance test schedules and test of independence. The first test verifies the null hypothesis that a frequency distribution is uniform (i.e. all entries or classes in the distribution have equal frequencies) against the alternative hypothesis that at least one is different. The test rejects the null hypothesis if the $\mathrm{p}$ value of significance is less than or equal to 0.05 . The second test verifies the null hypothesis based on the cross tabulation (contingency tables) that the two features are independent, against the alternative hypothesis that the features are dependent. The test rejects the null hypothesis if the $p$ value level of significance is less than or equal to 0.05 . The strength of association between variables was determined by the ratio V - Cramer and Pearson's contingency coefficient. The values of both of these factors vary from 0 (no association) to 1 (complete dependence). The level of statistical significance was considered to be $\mathrm{p}$-value $\leq 0.05$.

\section{Results}

\section{Knowledge about HT}

Almost half of the adolescents questioned in this survey (49.2\%) reveal a low level of global knowledge about HT. Nearly $38 \%$ of them have medium knowledge about it and only $13 \%$ have such knowledge on a good level (chi square $=50.65, \mathrm{df}=2, \mathrm{p} \leq 0.05$ ). Knowledge of its epidemiology, ways of treatment and prevention was not satisfactory either. Most of the adolescents have good knowledge only about the causes of HT (see Table 2).

Analysis results concerning the relationships between the level of global knowledge about HT among adolescents and demographic, environmental and medical factors are presented in Table 3. Children from urban areas revealed better knowledge than their peers from rural regions $(\mathrm{p}=0.007)$. Thorough analyses were devoted to the level of global and partial knowledge about HT in reference to the educational stages. Although slightly below statistical significance $(\mathrm{p}=0.08)$, a relationship between the level of global HT knowledge and the level of education was found. On the other hand, a significant relationship has been shown between the level of
Table 2 The level of partial knowledge about HT among adolescents

\begin{tabular}{|c|c|c|c|c|c|}
\hline \multirow{2}{*}{$\begin{array}{l}\text { Type of knowledge } \\
\text { about HT } \\
\text { Epidemiology }\end{array}$} & \multicolumn{2}{|l|}{ Level of knowledge } & \multicolumn{2}{|c|}{$\begin{array}{l}\text { Weak Medium } \\
n=250\end{array}$} & Good \\
\hline & Chi square $=77.86$ & $n$ & 144 & 75 & 31 \\
\hline & $d f=2$ & $\%$ & 57.6 & 30.0 & 12.4 \\
\hline & $p=0.0000$ & & & & \\
\hline \multirow[t]{3}{*}{ Causes } & Chi square $t=28.38$ & $\mathrm{n}$ & 65 & 62 & 123 \\
\hline & $d f=2$ & $\%$ & 26.0 & 24.8 & 49.2 \\
\hline & $p=0.0000$ & & & & \\
\hline \multirow[t]{3}{*}{ Symptoms } & Chi square $=173.86$ & $\mathrm{n}$ & 181 & 44 & 25 \\
\hline & $d f=2$ & $\%$ & 72.4 & 17.6 & 10.0 \\
\hline & $p=\mathbf{0 . 0 0 0 0}$ & & & & \\
\hline \multirow[t]{3}{*}{ Complications } & Chi square $=62.05$ & $\mathrm{n}$ & 94 & 128 & 28 \\
\hline & $d f=2$ & $\%$ & 37.6 & 51.2 & 11.2 \\
\hline & $p=\mathbf{0 . 0 0 0 0}$ & & & & \\
\hline \multirow[t]{3}{*}{ Ways of treatment } & Chi square $=7.4$ & $\mathrm{n}$ & 100 & 85 & 65 \\
\hline & $d f=2$ & $\%$ & 40.0 & 34.0 & 26.0 \\
\hline & $p=0.02$ & & & & \\
\hline \multirow[t]{3}{*}{ Prevention } & Chi square $=12.2$ & $\mathrm{n}$ & 105 & 85 & 60 \\
\hline & $d f=2$ & $\%$ & 42.0 & 34.0 & 24.0 \\
\hline & $p=\mathbf{0 . 0 0 2}$ & & & & \\
\hline
\end{tabular}

HT - hypertension.

education and the level of knowledge about HT complications $(\mathrm{p}=0.01)$ and the ways of its treatment $(p=0.002)$. It was also noted that the higher the school grade, the higher the percentage of children with better knowledge about HT.

Our results show that there is a statistically significant positive relation between the youths' global knowledge about HT and family history of HT $(\mathrm{p}=0.01)$. The global level of knowledge of HT depending on its source is presented in Table 3. It was found that when school is taken as the main source of global HT knowledge, its level is relatively low. A similar analogy can be observed when referring to the level of knowledge about symptoms, complications and HT epidemiology. Among the people who named their families as the main source of such knowledge, there is a significant majority revealing poor global knowledge (either weak or medium). The youth also revealed a weak level of knowledge about HT epidemiology and symptoms. A good level of knowledge was found only in the causes of HT. No significant relations between the level of global knowledge about HT and the Internet $(\mathrm{p}=0.13)$, the radio, newspapers or TV $(\mathrm{p}=0.33)$ as a source of knowledge were found.

\section{Subjective factors}

The analysis results regarding the level of knowledge about HT and subjective factors are presented in Tables 4 
Table 3 The level of global knowledge about HT among adolescents and the chosen demographic, environmental and medical factors

\begin{tabular}{|c|c|c|c|c|c|}
\hline Factor & Level of knowledge & & Weak & Medium & Good \\
\hline Sex & & & & $n=249$ & \\
\hline \multirow[t]{2}{*}{ Girls } & Chi square $=0.01$ & $n$ & 71 & 54 & 19 \\
\hline & $d f=2$ & $\%$ & 49.3 & 37.5 & 13.2 \\
\hline \multirow[t]{2}{*}{ Boys } & & $\mathrm{n}$ & 51 & 40 & 14 \\
\hline & $p=1$ & $\%$ & 48.6 & 38.1 & 13.3 \\
\hline Place of living & & & & $n=247$ & \\
\hline \multirow[t]{2}{*}{ Urban } & Chi square $=9.77$ & $\mathrm{n}$ & 36 & 46 & 16 \\
\hline & $\mathrm{df}=2$ & $\%$ & 36.7 & 46.9 & 16.3 \\
\hline \multirow[t]{2}{*}{ Rural } & & $\mathrm{n}$ & 85 & 47 & 17 \\
\hline & $p=\mathbf{0 . 0 0 7}$ & $\%$ & 57.0 & 31.5 & 11.4 \\
\hline Level of education & & & & $n=249$ & \\
\hline \multirow[t]{2}{*}{ Grade I } & Chi square $=8.46$ & $\mathrm{n}$ & 41 & 27 & 6 \\
\hline & & $\%$ & 55.41 & 36.49 & 8.11 \\
\hline \multirow[t]{2}{*}{ Grade II } & $d f=4$ & $\mathrm{n}$ & 65 & 52 & 16 \\
\hline & & $\%$ & 48.87 & 39.10 & 12.03 \\
\hline \multirow[t]{2}{*}{ Grade III } & $p=0.08$ & $n$ & 17 & 14 & 11 \\
\hline & & $\%$ & 40.48 & 33.33 & 26.19 \\
\hline Blood pressure tests in the past & & & & $n=246$ & \\
\hline \multirow[t]{2}{*}{ No } & Chi square $=14.63$ & $\mathrm{n}$ & 21 & 4 & 0 \\
\hline & $\mathrm{df}=2$ & $\%$ & 84.0 & 16.0 & 0.0 \\
\hline \multirow[t]{2}{*}{ Yes } & & $n$ & 98 & 90 & 33 \\
\hline & $p=0.0006$ & $\%$ & 44.3 & 40.7 & 14.9 \\
\hline HT family history & & & & $n=250$ & \\
\hline \multirow[t]{2}{*}{ No } & Chi square $=8.98$ & $n$ & 67 & 39 & 9 \\
\hline & $d f=2$ & $\%$ & 58.26 & 33.91 & 7.83 \\
\hline \multirow[t]{2}{*}{ Yes } & & $\mathrm{n}$ & 56 & 55 & 24 \\
\hline & $p=0.01$ & $\%$ & 41.48 & 40.74 & 17.78 \\
\hline Other chronic diseases in family & & & & $n=250$ & \\
\hline \multirow[t]{2}{*}{ No } & Chi square $=1.35$ & $n$ & 66 & 47 & 14 \\
\hline & $\mathrm{df}=2$ & $\%$ & 52.0 & 37.0 & 11.0 \\
\hline \multirow[t]{2}{*}{ Yes } & & $\mathrm{n}$ & 57 & 47 & 19 \\
\hline & $p=0.51$ & $\%$ & 46.3 & 38.2 & 15.4 \\
\hline Source of knowledge about HT & & & & $n=171$ & \\
\hline \multirow[t]{3}{*}{ School } & Chi square $=20.95$ & $n$ & 74 & 68 & 29 \\
\hline & $d f=2$ & $\%$ & 43.3 & 39.8 & 17.0 \\
\hline & $p=\mathbf{0 . 0 0 0 0}$ & & & & \\
\hline \multirow[t]{3}{*}{ Family } & Chi square $=18.77$ & $n$ & 46 & 55 & 18 \\
\hline & $\mathrm{df}=2$ & $\%$ & 38.7 & 46.2 & 15.1 \\
\hline & $p=\mathbf{0 . 0 0 0 0}$ & & & & \\
\hline \multirow[t]{3}{*}{ Internet } & Chi square $=4.12$ & $n$ & 30 & 35 & 20 \\
\hline & $\mathrm{df}=2$ & $\%$ & 35.3 & 41.2 & 23.5 \\
\hline & $p=0.13$ & & & & \\
\hline
\end{tabular}


Table 3 The level of global knowledge about HT among adolescents and the chosen demographic, environmental and medical factors (Continued)

\begin{tabular}{llllll}
\hline Radio/TV/newspapers & C Chi square $=2.21$ & $n$ & 20 & 23 & 40.4 \\
& $\mathrm{df}=2$ & $\%$ & 35.1 & 24.6 \\
$\mathrm{p}=0.33$ & & & \\
\hline
\end{tabular}

HT - hypertension.

and 5 . It has been shown that there was no statistically significant association between the level of global knowledge about HT, the level of knowledge about its symptoms, complications, ways of treatment and prevention and the ranges of the applied category of "good health" as the symbol of happiness. However, it was noticed that there is a tendency to display better global knowledge by the people who choose "good health" as an important symbol of happiness. Nevertheless, there is a statistically significant relationship $(\mathrm{p}=0.05)$ between the level of knowledge about HT causes and the value applied to the category of "good health". Taking all the people with good knowledge about HT causes into consideration, only $2.5 \%$ of them do not recognize "good health" as an important symbol of happiness. In the group of the people with weak and medium knowledge on this issue, the percentage mentioned above is, respectively, $14.75 \%$ and $13.11 \%$. The precise data is presented in Table 4 .

It was observed that there is no significant relationship between the level of knowledge about HT, both on a global scale and in the aspects of its individual components, and considering the category of "good health, physical and mental efficiency" an important personal value. Similarly, it was noted that there is no significant association between the level of global knowledge about HT and a feeling of one's own personal competences (see Table 5). It was also noted that people with a stronger feeling of strength possess vividly statistically better knowledge than the ones with a weaker feeling of strength $(p=0.006)$. Such a relationship was not observed when referring to a feeling of persistence. It was noticed as well that the percentage of people with a higher feeling of strength and good knowledge about HT treatment is much higher (31.6\%) than the percentage of the youth with a lower feeling of strength and good level of knowledge $(\mathrm{p}=0.05)$.

\section{Discussion}

The results of our study have shown that the youths' knowledge about HT is both unsatisfactory and random, which indicates the necessity of its promotion across this age group, especially in the field of its symptoms. According to the research performed by various authors, general knowledge among young people, when applied to noninfectious diseases, is very poor [16,17]. Among others, Vanhecke et al. [18] demonstrated that adolescents lack knowledge regarding the risk of cardiovascular disease and this consequently corresponds with our own observations as far as the issue of HT is concerned.

The research has not revealed any significant relationship between the youths' knowledge about HT and their age. However, the higher the level of education, the higher the percentage is of adolescents presenting good knowledge about this disease, whereas the percentage of young people with poor knowledge about it decreases. Such a tendency is in accordance with a commonlyknown concept claiming that children's knowledge expands along with increasing years of education [19-21].

The results of our own research do not show any differences in global knowledge about HT when taking sex into consideration. As for other authors, their observations in this field are varied $[18,22,23]$. Our research has also shown that urban adolescents have better knowledge about HT than their peers from rural areas. The explanation of these results lies in the diversification of learning progress, which is varied for children of different regions and different social backgrounds [24]. Adolescents of rural areas tend to obtain lower rates in an intellectual learning process. Therefore, during educational activities, it is worth paying attention to the fact that the acquisition of knowledge about health and diseases might become more difficult for such groups of students.

School is reported to be perceived by the youth as the main source of knowledge about HT. This is why such a low level of global knowledge about this issue is alarming, as it indicates certain teaching shortcomings in educational institutions. Our research has also revealed the fact that a family is not thought to be a source of a good knowledge about HT either. Such an unsatisfactory level of knowledge indicates that, despite their sources, all the health messages concerning HT show visible weaknesses. It might be connected with their content, form and ways of transmission or ultimately their inadequate adaptation to a specific group of receivers. All of the factors shown above indicate the need for modification of both the content and forms of educational undertakings.

The current study has revealed as well that the students who previously had had blood pressure measurements possess twice the degree of knowledge about HT than their peers who have not had such tests so far. In fact, none of this group is reported to have a good a level of knowledge about it. The results imply the necessity of conducting blood pressure tests during check-ups in 
Table 4 The level of global and partial knowledge about HT according to choosing "good health" as a symbol of happiness among adolescents $(n=242)$

\begin{tabular}{|c|c|c|c|c|c|c|c|c|}
\hline Level of knowledge about HT & Ranges & & 0 & 1 & 2 & 3 & 4 & 5 \\
\hline Epidemiological knowledge about HT & weak & $\mathrm{n}$ & 14 & 20 & 14 & 21 & 27 & 41 \\
\hline Contingency coefficient $C=0.21$ & & $\%$ & 10.22 & 14.60 & 10.22 & 15.33 & 19.71 & 29.93 \\
\hline Cramer coefficient $V=0.15$ & medium & $\mathrm{n}$ & 5 & 4 & 6 & 15 & 19 & 25 \\
\hline Chi square $=11.44$ & & $\%$ & 6.76 & 5.41 & 8.11 & 20.27 & 25.68 & 33.78 \\
\hline$d f=10$ & good & $\mathrm{n}$ & 1 & 2 & 5 & 3 & 10 & 10 \\
\hline$p=0.32$ & & $\%$ & 3.23 & 6.45 & 16.13 & 9.68 & 32.26 & 32.26 \\
\hline Knowledge about HT causes & weak & $\mathrm{n}$ & 9 & 3 & 4 & 11 & 16 & 18 \\
\hline Contingency coefficient $C=0.26$ & & $\%$ & 14.75 & 4.92 & 6.56 & 18.03 & 26.23 & 29.51 \\
\hline Cramer coefficient $V=0.19$ & medium & $\mathrm{n}$ & 8 & 10 & 7 & 5 & 13 & 18 \\
\hline Chi square $=18.3841$ & & $\%$ & 13.11 & 16.39 & 11.48 & 8.20 & 21.31 & 29.51 \\
\hline$d f=10$ & good & $n$ & 3 & 13 & 14 & 23 & 27 & 40 \\
\hline$p=0.05$ & & $\%$ & 2.50 & 10.83 & 11.67 & 19.17 & 22.50 & 33.33 \\
\hline Knowledge about HT symptoms & weak & $\mathrm{n}$ & 15 & 23 & 20 & 28 & 39 & 51 \\
\hline Contingency coefficient $C=0.19$ & & $\%$ & 8.52 & 13.07 & 11.36 & 15.91 & 22.16 & 28.29 \\
\hline Cramer coefficient V $=0.13$ & medium & $n$ & 4 & 1 & 4 & 8 & 11 & 13 \\
\hline Chi square $=8.94$ & & $\%$ & 9.76 & 2.44 & 9.76 & 19.51 & 26.83 & 31.71 \\
\hline$d f=10$ & good & $n$ & 1 & 2 & 1 & 3 & 6 & 12 \\
\hline$p=0.54$ & & $\%$ & 4.00 & 8.00 & 4.00 & 12.00 & 24.00 & 48.00 \\
\hline Knowledge about HT complications & weak & $\mathrm{n}$ & 10 & 12 & 11 & 14 & 21 & 21 \\
\hline Contingency coefficient $C=0.2$ & & $\%$ & 11.24 & 13.48 & 12.36 & 15.73 & 23.60 & 23.60 \\
\hline Cramer coefficient $V=0.14$ & medium & $\mathrm{n}$ & 10 & 11 & 12 & 19 & 26 & 47 \\
\hline Chi square $=10.21$ & & $\%$ & 8.00 & 8.80 & 9.60 & 15.20 & 20.80 & 37.60 \\
\hline$d f=10$ & good & $\mathrm{n}$ & 0 & 3 & 2 & 6 & 9 & 8 \\
\hline$p=0.42$ & & $\%$ & 0.00 & 10.71 & 7.14 & 21.43 & 32.14 & 28.57 \\
\hline Knowledge about HT treatment & weak & $n$ & 8 & 9 & 9 & 16 & 23 & 30 \\
\hline Contingency coefficient $C=0.12$ & & $\%$ & 8.42 & 9.47 & 9.47 & 16.84 & 24.21 & 31.58 \\
\hline Cramer coefficient V $=0.08$ & medium & $\mathrm{n}$ & 8 & 10 & 8 & 14 & 21 & 22 \\
\hline Chi square $=3.54$ & & $\%$ & 9.64 & 12.05 & 9.64 & 16.87 & 25.30 & 26.51 \\
\hline$d f=10$ & good & $\mathrm{n}$ & 4 & 7 & 8 & 9 & 12 & 24 \\
\hline$p=0.96$ & & $\%$ & 6.25 & 10.94 & 12.50 & 14.06 & 18.75 & 37.50 \\
\hline Knowledge about HT prevention & weak & $n$ & 7 & 13 & 11 & 21 & 17 & 30 \\
\hline Contingency coefficient $C=0.18$ & & $\%$ & 7.07 & 13.13 & 11.11 & 21.21 & 17.17 & 30.30 \\
\hline Cramer coefficient $V=0.13$ & medium & $n$ & 9 & 6 & 9 & 9 & 23 & 27 \\
\hline Chi square $=8.6$ & & $\%$ & 10.84 & 7.23 & 10.84 & 10.84 & 27.71 & 32.53 \\
\hline$d f=10$ & good & $n$ & 4 & 7 & 5 & 9 & 16 & 19 \\
\hline$p=0.57$ & & $\%$ & 6.67 & 11.67 & 8.33 & 15.00 & 26.67 & 31.67 \\
\hline Global knowledge about HT & weak & $n$ & 11 & 16 & 13 & 20 & 25 & 31 \\
\hline Contingency coefficient $C=0.2$ & & $\%$ & 9.48 & 13.79 & 11.21 & 17.24 & 21.55 & 26.72 \\
\hline Cramer coefficient $V=0.14$ & medium & $n$ & 9 & 8 & 10 & 13 & 20 & 34 \\
\hline Chi square $=9.83$ & & $\%$ & 9.57 & 8.51 & 10.64 & 13.83 & 21.28 & 36.17 \\
\hline$d f=10$ & good & $\mathrm{n}$ & 0 & 2 & 2 & 6 & 11 & 11 \\
\hline$p=0.45$ & & $\%$ & 0.00 & 6.25 & 6.25 & 18.75 & 34.38 & 34.38 \\
\hline
\end{tabular}

HT - hypertension. 
Table 5 Results of personal competence scale among adolescents and global knowledge about HT

\begin{tabular}{|c|c|c|c|c|c|}
\hline \multirow[t]{2}{*}{ Personal competence scale } & & & \multicolumn{3}{|c|}{ Global knowledge about HT } \\
\hline & & & Weak & Medium & Good \\
\hline Feeling of self-effectiveness (PWS) $(n=237)$ & $1-4$ & $\mathrm{n}$ & 26 & 16 & 3 \\
\hline Contingency coefficient $C=0.18$ & low & $\%$ & 57.78 & 35.56 & 6.67 \\
\hline Cramer coefficient V $=0.13$ & $5-6$ & $\mathrm{n}$ & 49 & 29 & 12 \\
\hline Chi square $=8.19$ & medium & $\%$ & 54.44 & 32.22 & 13.33 \\
\hline$d f=4$ & $7-10$ & $\mathrm{n}$ & 39 & 46 & 17 \\
\hline$p=0.08$ & high & $\%$ & 38.24 & 45.10 & 16.67 \\
\hline Feeling of strength $(S)(n=238)$ & $1-18$ & $\mathrm{n}$ & 61 & 39 & 7 \\
\hline Contingency coefficient $C=0.2$ & lower & $\%$ & 57.01 & 36.45 & 6.54 \\
\hline \multicolumn{6}{|l|}{ Cramer coefficient $V=0.2$} \\
\hline Chi square $=10.09$ & $19-24$ & $\mathrm{n}$ & 54 & 52 & 25 \\
\hline$d f=2$ & higher & $\%$ & 41.22 & 39.69 & 19.08 \\
\hline \multicolumn{6}{|l|}{$p=0.006$} \\
\hline Feeling of persistence $(W)(n=241)$ & $1-16$ & $\mathrm{n}$ & 59 & 39 & 20 \\
\hline Contingency coefficient $C=0.12$ & lower & $\%$ & 50.0 & 33.1 & 16.9 \\
\hline \multicolumn{6}{|l|}{ Cramer coefficient $V=0.12$} \\
\hline Chi square $=3.55$ & $17-24$ & $n$ & 57 & 53 & 13 \\
\hline$d f=2$ & higher & $\%$ & 46.3 & 43.1 & 10.6 \\
\hline$p=0.17$ & & & & & \\
\hline
\end{tabular}

medical centers and putting practice and theory on HT into one.

It is known that an individual's knowledge is best put into practice only if the subject of knowledge applies to the system of a given person's value. The results of our study show that $20 \%$ of young people fail to consider health to be a component of their system of important personal values. On the other hand, almost one in three of the surveyed youth admitted that health is the most important symbol of happiness. According to our own analysis as well as the opinions given by healthcare experts, young people do not consider health a value itself but treat it rather as a way to obtain happiness in their lives. Such an approach may lead to undertaking activities which might cause harm to their health [24]. The perception that health is merely a product to be purchased brings passiveness and a lack of remorse and motivation to actions whose aim is to both maintain and strengthen their health condition. It also causes a lack of responsibility for their health and lifestyles. This is why any educational activities should include making adolescents more aware of the value of health in their lives. Such consciousness may influence their feelings of the realization of their health and treating their health selfcare in terms of a necessity, not only an obligation.

The starting of a specific activity by a person depends on one's beliefs about one's ability to influence the course of action. In our study, four out of ten of those surveyed reported a feeling of high self-effectiveness, and nearly two in ten revealed low competence markers. In spite of the fact that no significant association was observed between a feeling of self-effectiveness among students and their knowledge about HT, we can see a tendency among the youth to have better knowledge about HT only if it is accompanied by an increase in selfeffectiveness. The relatively high self-efficacy in the examined group of adolescents can be considered a positive factor in the prevention of youth health issues. It has a positive effect on the processing of information, formulating goals, undertaking tasks and their execution [25,26]. A sense of one's own self-efficacy will therefore be important for the implementation of preventive educational programs.

The conducted study has some limitations. First, the effective sample size of the study, although selected from among a large number of secondary school students, is small, implying a limited statistical power. Our sample was derived from one region of Poland, therefore the observations captured herein can only be generalized to settings with a similar context. However, it is likely that similar processes exist in other parts of Poland and the results of this study could be used as a basis for a larger survey which could attempt to confirm them in a broader setting.

In our study, we analyzed, among others, the association between the level of knowledge about HT among adolescents and their system of values, including health. Taking into account different systems of values could 
show other results. Although all of the students completed the survey unassisted in their schools, we can't exclude the impact of location and context on choices made. Finally, we did not analyze many other factors that may be related to the knowledge of HT and may be useful in designing preventive strategies in an adolescent population, so further studies are needed in this area.

\section{Conclusions}

Knowledge about HT among adolescents remains unsatisfactory and random, which indicates the necessity for routine education in this field, especially as it applies to HT symptoms. It seems that the consideration such elements as blood pressure measurement and family history of HT in education programs can improve their efficiency. Particular attention should be paid to the strengthening of "health" as a personal value and to build young people's sense of personal competence for the creation and implementation of healthy behavior.

\section{Additional file}

Additional file 1: The authors' questionnaire.

\section{Abbreviation}

HT: Hypertension; PHT: Primary hypertension; BP: Blood pressure.

\section{Competing interests}

The authors declare that they have no competing interests.

\section{Authors' contributions}

IG participated in the study design, performed data collection and analysis and interpretation of data, and contributed to the drafts of the manuscript. AMM contributed to the interpretation of the data, and has been involved in revising the manuscript critically for important intellectual content. KKP was responsible for the idea and design of the study, contributed to the analysis and interpretation of the data, and for the drafts and the final manuscript. All authors have read and approved the final manuscript.

\section{Author details}

${ }^{1}$ Department of Nursing, Public Medical Academy, Opole, Poland.

${ }^{2}$ Department of Family Medicine, Wroclaw Medical University, Wroclaw, Poland. ${ }^{3}$ Department of Pediatric Nephrology, Wroclaw Medical University, Wroclaw, Poland.

Received: 20 November 2014 Accepted: 22 April 2015

Published online: 03 May 2015

\section{References}

1. Kearney PM, Whelton M, Reynolds K, Muntner P, Whelton PK, He J. Global burden of hypertension: analysis of worldwide data. Lancet. 2005;365:217-23.

2. Akgun C, Dogan M, Akbayram S, Tuncer O, Peker E, Taskin G, et al. The incidence of asymptomatic hypertension in school children. J Nippon Med Sch. 2010;77:160-5.

3. Falkner B, Lurbe E, Schaefer F. High Blood pressure in children: clinical and health policy implications. J Clin Hypertens. 2010;12:261-76.

4. Falkner B, Gidding S. Childhood Obesity and Blood Pressure. Back to the Future? Hypertension. 2011;58:754-5.

5. Lurbe E: Hypertension in children and adolescents. In Manual of Hypertension of the European Society of Hypertension. Edited by Mancia G, Grassi G, Kjeldsen S. Informa Healthcare; 2008:336-346.

6. Kilis-Pstrusinska K, Zwolinska D. Primary hypertension as a growing problem in pediatrics. Fam Med Prim Care Rev. 2007:9:809-14.
7. Falkner B. Hypertension in children and adolescents: epidemiology and natural history. Pediatr Nephrol. 2010;25:1219-24.

8. The Diagnosis and Management of Hypertension in Children. Can Hypertens Soc Bull 2008; 95:1-8.

9. National High Blood Pressure Education Program Working Group on High Blood Pressure in Children and Adolescents. The Fourth Report on the Diagnosis, Evaluation, and Treatment of High Blood Pressure in Children and Adolescents. Pediatrics. 2004;114:555-75.

10. Litwin M. Primary hypertension in children and adolescents - pathophysiology. In: Litwin M, Januszewicz A, Prejbisz A, editors. Hypertension in adolescents and young adults. Krakow: Practical Medicine; 2011. p. 289-316.

11. Hayman LL, Meininger JC, Daniels SR, McCrindle BW, Helden L, Ross J, et al. Primary prevention of cardiovascular disease in nursing practice: focus on children and youth. Circulation. 2007;116:344-57.

12. Juresa V. Preventive programmes in school health care services. In: Donev D, Pavlekowic G, Zalatel Kragelij L, editors. Health promotion and disease prevention. A handbook for teachers, researchers, health professionals and decision makers. Skopje: Hans Jacobs Publishing Company; 2007. p. 396-405.

13. Darnton-Hill I, Nishida C, James WP. A life course approach to diet, nutrition and the prevention of chronic diseases. Public Health Nutr. 2004;7:101-2.

14. Litwin M, Niemirska A. Organ damage in children hypertension. In: Litwin M, Januszewicz A, Prejbisz A, editors. Hypertension in adolescents and young adults. Krakow: Practical Medicine; 2011. p. 241-69.

15. Juczyński Z. Measurement tool in health promotion and psychology. Warszawa: Laboratory of Psychological Tests; 2009.

16. Lynch EB, Liu K, Kiefe Cl, Greenland P. Cardiovascular disease risk factor knowledge in young adults and 10-year change in risk factors: the Coronary Artery Risk Development in Young Adults (CARDIA) Study. Am J Epidemiol. 2006;164:1171-9.

17. Vale A. Heart disease and young adults: Is prevention important? J Community Health Nurs. 2000;17:225-33.

18. Vanhecke TE, Miller WM, Franklin BA, Weber JE, McCullough PA. Awareness, knowledge and perception of heart disease among adolescents. Eur J Cardiovasc Prev Rehabil. 2006;13:718-23.

19. Card D. The causal effect of education on earnings. In Handbook of Labor Economics. Edited by Ashenfelter O, Card D. Amsterdam. 1999. 1802-1859.

20. Lleras-Muney A. The Relationship between Education and Adult Mortality in the United States. Rev Econ Stud. 2005;72:189-221.

21. Spasojevic J. Effects of Education on Adult Health in Sweden: Results from Natural Experiment. PD thesis. City University of New York, Graduate Center, 2003.

22. Niknian M, Mckinlay SM, Rakowski W, Carleton RA. A comparison of perceived and objective cvd risk in a general population. Am J Public Health. 1989;79:1653-4.

23. Winham DM, Jones KM. Knowledge of young African American adults about heart disease: a cross-sectional survey. BMC Public Health. 2011;11:248. doi:10.1186/1471-2458-11-248.

24. Zadworna-Cieślak M, Ogińska-Bulik N. Adolescent health behaviors - symptoms and family circumstances. Warszawa: Diffin; 2011.

25. Bandura A, Barbaranelli C, Caprara GV, Pastorelli C. Self-efficacy beliefs as shapers of children's aspirations and career trajectories. Child Dev. 2001;72:187-206.

26. Kościelak R. Locus of control and self-efficacy in health and disease. Krakow: Publishing House "Pulse"; 2010.

\section{Submit your next manuscript to BioMed Central and take full advantage of:}

- Convenient online submission

- Thorough peer review

- No space constraints or color figure charges

- Immediate publication on acceptance

- Inclusion in PubMed, CAS, Scopus and Google Scholar

- Research which is freely available for redistribution 